- Raises awareness of acute leukaemia as a possible cause of gingival enlargement.

- Increases awareness of differential diagnosis of gingival enlargement.

- Highlights the importance of collaboration between specialties.

- Emphasises the importance of considering general medical conditions as a cause for dental problems.

\title{
Gingival infiltration in acute monoblastic leukaemia
}

\author{
P. Gallipoli and M. Leach²
}

\begin{abstract}
Acute myeloid leukaemias (AML) are aggressive haematopoietic neoplasms that, if untreated, can lead to death within days. Up to $40 \%$ of presenting patients show evidence of extramedullary involvement (EMI) at diagnosis. EMI is reportedly most prevalent in myelo-monoblastic and monoblastic subtypes of AML (M4 and M5 according to FAB classification) and can present as leukaemic infiltrates in many sites including gingival enlargement and mucosal and skin nodules. We report a case of a patient who presented with gingival enlargement secondary to leukaemic infiltration. This case shows the importance of awareness of leukaemic infiltration as a cause for gingival enlargement.
\end{abstract}

\section{CASE REPORT}

A 45-year-old Caucasian woman, with a history of rheumatoid arthritis, presented with a four week history of lethargy, night sweats and painful and enlarged gingivae. She was seen by a dentist and then her general medical practitioner and originally treated with oral antibiotics for presumed oral infection. Her condition deteriorated and she presented to hospital. The admitting physicians also noted the marked gingival enlargement (Figs 1-2) but did not appreciate its significance. Full blood count on admission showed $\mathrm{Hb} 82 \mathrm{~g} / \mathrm{L}$, WBC $31 \times 10^{9} / \mathrm{L}$ and platelets $81 \times 10^{9} / \mathrm{L}$. Acute monoblastic leukaemia was suspected clinically and this was confirmed

${ }^{1}$ Haematology SpR, West of Scotland Deanery: ${ }^{2}$ Consultant Haematologist, Stobhill Hospital, 133 Balornock Road, Glasgow, G21 3UT

*Correspondence to: Dr Paolo Gallipoli

Email:p_gallipoli@hotmail.com

\section{Refereed Paper}

Accepted 11 June 2007

DOI: $10.1038 /$ bdj.2007.994

${ }^{\circ}$ British Dental Journal 2007; 203: 507-509 on blood and bone marrow morphology (Fig. 3) and flow cytometry. The gingival enlargement improved rapidly on commencing induction chemotherapy with daunorubicin, cytarabine and etoposide. This treatment was successful in achieving complete remission in the bone marrow. The patient has now completed four courses of chemotherapy. She remains in remission 18 months after diagnosis and the gingival enlargement and pain has resolved completely.

\section{DISCUSSION}

Acute onset gingival enlargement is an unusual physical sign with a narrow differential diagnosis. When approaching a patient with gingival enlargement, a primary care practitioner should initially elucidate if the enlargement is old and chronic or new. Recent onset gingival enlargement can be secondary to a local cause, systemic illness or a drug reaction. A detailed drug history is paramount as the most common cause of recent onset gingival enlargement is drug therapy, having been reported with the use of phenytoin, ${ }^{1}$ calcium channel blockers, ${ }^{2}$ and cyclosporine. ${ }^{3}$ Chronic gingivitis mainly secondary to poor oral hygiene is a common local cause of gingival enlargement. Systemic illnesses such as Wegener's granulomatosis, sarcoidosis, Crohn's disease and acromegaly ${ }^{4}$ have also been described in association with gingival enlargement and signs and symptoms of these conditions must be actively sought when approaching such patients. AML is the most serious condition associated with gingival enlargement. In patients with AML other signs and symptoms of bone marrow failure such as mucosal ecchymoses and gingival bleeding and systemic symptoms such as night sweats, recent infections and lethargy can accompany the gingival enlargement. A full blood count $(\mathrm{FBC})$ is a simple and useful test in these circumstances and can help to reach a prompt diagnosis. In our case failure to recognise the significance of the systemic symptoms led to a delay in the diagnosis that was potentially life-threatening for the patient.

Gingival infiltration is a well known feature of the acute myeloid leukaemias but particularly those of myelomonoblastic and monoblastic lineages; 

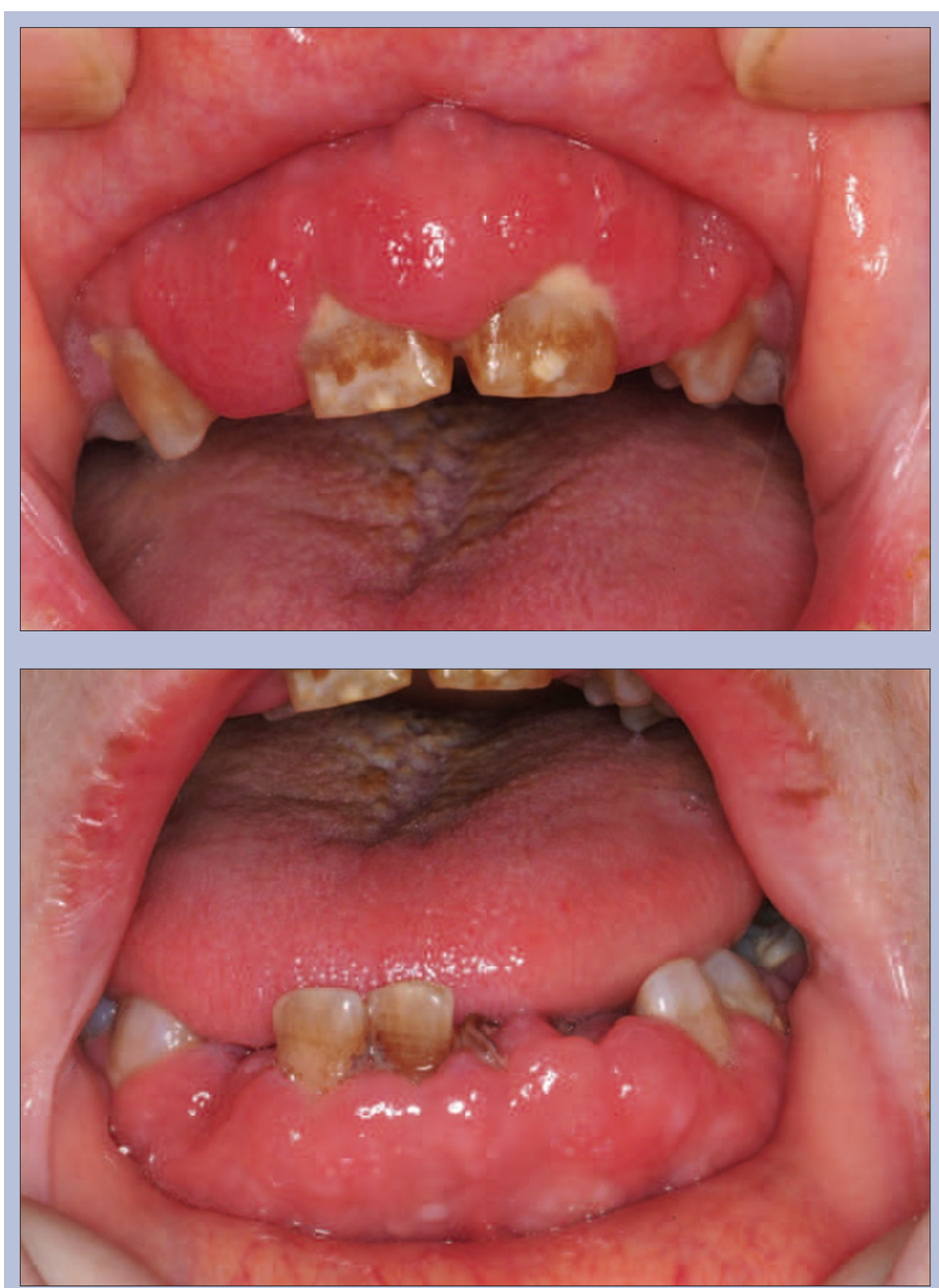

Figs 1-2 Marked gingival enlargement affecting both upper and lower gingivae in our patient affected by acute monoblastic leukaemia

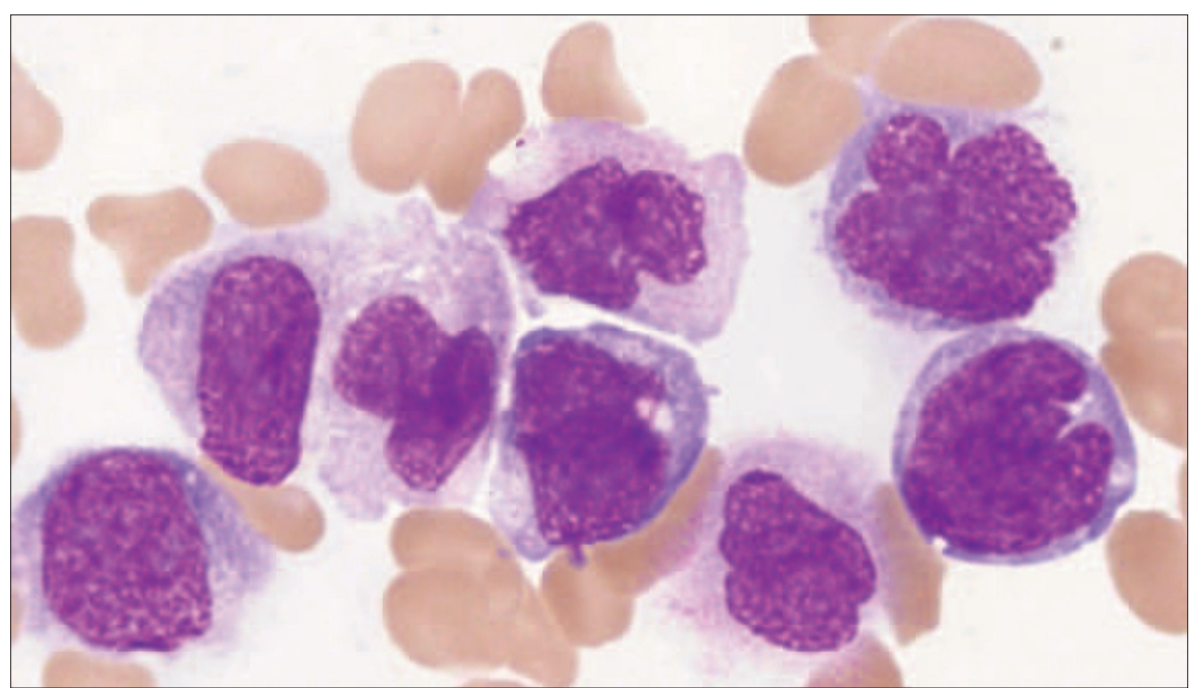

Fig. 3 Bone marrow aspirate morphology from our patient affected by acute monoblastic leukaemia showing a monomorphic population of monoblasts subtypes M4 and M5 according to the French American British (FAB) classification. It is present in about 30\% of patients at diagnosis with these AML subtypes. ${ }^{5}$ These patients tend to present with a high white blood cell count and may also have skin infiltrates, meningeal infiltrates or hepatosplenomegaly secondary to leukaemic blasts infiltration. Extramedullary involvement is associated with CD56 expression on blast cells and karyotypic abnormalities involving chromosome 11, in particular abnormalities of 11q23 involving the MLL gene. ${ }^{5-7}$ Our patient's cells, however, had normal cytogenetics and CD56 (a natural killer cell marker) was not aberrantly expressed.

AML is the most common myeloid leukaemia with a prevalence of 3.8 cases per 100,000 with a median age at presentation of 70 years. It is a clonal disorder of haematopoietic stem cells. The disease causes a rapid onset of bone marrow failure causing anaemia, neutropenia and thrombocytopenia. Most cases arise in individuals without antecedent blood disorders but there is an increasing incidence of AML arising from myelodysplastic syndromes and as a result of prior chemotherapy used to treat solid tumours. In patients younger than 60 years of age, cytotoxic chemotherapy might cure $20-75 \%$ of cases, according primarily to prognostic risk groups attributed to the type of cytogenetic abnormality seen in the leukaemic cells. ${ }^{8}$ Patients with AML who present with EMI at diagnosis, seem to have a lower complete remission rate to induction chemotherapy and a lower overall survival rate. Therefore these patients may warrant an alternative therapeutic approach, including stem cell transplant, to improve their outcome. ${ }^{5-8}$

It is imperative that acute myeloid leukaemia is diagnosed promptly. Early diagnosis allows the introduction of specific chemotherapy but also supportive treatment with blood products and early treatment of infection with broad spectrum intravenous antibiotics. Many patients present with cutaneous bleeding or bruising but some develop life threatening gastrointestinal or intracerebral haemorrhage. Neutropenic fever is common. Septicaemia can be rapid in onset and may cause multiorgan failure before antibiotic therapy can be introduced. Early diagnosis of AML is therefore 
imperative and gingival enlargement may be an early physical sign to alert the clinician to this possibility.

1. Soga Y, Nishimura F, Ohtsuka Y et al. CYP2C polymorphisms, phenytoin metabolism and gingiva overgrowth in epileptic subjects. Life Sci 2004; 74: 827-834.

2. Samarasinghe $Y P_{1}, \operatorname{Cox} A$, Feher M D. Calcium channel blocker induced gum hypertrophy: no class distinction. Heart 2004; $90: 16$

3. Vescovi P, Meleti M Manfredi M, Bonanini M. Pathogenesis of cyclosporine induced gingival overgrowth. Minerva Stomatol 2003; 52: 219-229.

4. Khera P, Zirwas M J, English J C. Diffuse gingival enlargement. J Am Acad Dermato/ 2005; 52: 491-499.

5. Chang H, Brandwein J, Yi Q L, Chun K, Patterson $B$, Brien B. Extramedullary infiltrates of AML are associated with CD56 expression, 11q23 abnormalities and inferior clinical outcome. Leuk Res 2004; 28: 1007-1011.
6. Vural F, Ozcan M A, Ozsan G H et al. Gingival involvement in a patient with CD56+ chronic myelomonocytic leukaemia. Leuk Lymphoma 2004; 45: 415-418.

7. Blum W, Mrozek K, Ruppert A S et al. Adult de novo acute myeloid leukemia with $\mathrm{t}(6 ; 11)(q 27 ; q 23)$ : results from cancer and Leukemia Group B Study 8461 and review of the literature. Cancer 2004; 101: 1420-1427.

8. Estey E, Dohner H. Acute Myeloid Leukaemia. Lancet 2006; 368: 1894-1907. 